\title{
A Decision Rule to Minimize Daily Capital Charges \\ in Forecasting Value-at-Risk*
}

\author{
Michael McAleer \\ Department of Quantitative Economics \\ Complutense University of Madrid \\ and \\ Econometric Institute \\ Erasmus University Rotterdam
}

Juan-Angel Jimenez-Martin

Department of Quantitative Economics

Complutense University of Madrid

\section{Teodosio Pérez-Amaral}

Department of Quantitative Economics

Complutense University of Madrid

February 2009

* This paper was written while the first author was visiting the Econometric Institute, Erasmus School of Economics, Erasmus University Rotterdam, in autumn 2008. The first author wishes to thank the Australian Research Council for financial support, and the Erasmus School of Economics for their gracious hospitality and excellent working environment. We wanted to thank Jesus Ruiz for his help with graphs. 


\begin{abstract}
Under the Basel II Accord, banks and other Authorized Deposit-taking Institutions (ADIs) have to communicate their daily risk estimates to the monetary authorities at the beginning of the trading day, using a variety of Value-at-Risk (VaR) models to measure risk. Sometimes the risk estimates communicated using these models are too high, thereby leading to large capital requirements and high capital costs. At other times, the risk estimates are too low, leading to excessive violations, so that realised losses are above the estimated risk. In this paper we propose a learning strategy that complements existing methods for calculating $\mathrm{VaR}$ and lowers daily capital requirements, while restricting the number of endogenous violations within the Basel II Accord penalty limits. We suggest a decision rule that responds to violations in a discrete and instantaneous manner, while adapting more slowly in periods of no violations. We apply the proposed strategy to Standard \& Poor's 500 Index and show there can be substantial savings in daily capital charges, while restricting the number of violations to within the Basel II penalty limits.
\end{abstract}

Key words and phrases: Daily capital charges, endogenous violations, frequency of violations, optimizing strategy, risk forecasts, value-at-risk.

JEL Classifications: G32, G11, G17, C53. 


\section{Introduction}

The Value-at-Risk (VaR) concept has become a standard tool in the exploding area of risk measurement and management. In brief, $\mathrm{VaR}$ is defined as an estimate of the probability and size of the potential loss to be expected over a given period. This concept has become especially important following the 1995 amendment to the Basel Accord, whereby banks and other Authorized Deposit-taking Institutions (ADIs) were permitted to use internal models to calculate their VaR thresholds (see Jorion (2000) for a detailed discussion of $\mathrm{VaR}$ ). Consequently, the last few years have witnessed a growing literature comparing modelling approaches and implementation procedures to answer the question of how to measure VaR, with many research studies arguing in favour or against various VaR models.

The amendment to the Basel Accord was designed to reward institutions with superior risk management systems. A back-testing procedure, whereby the realized returns are compared with the VaR forecasts, was introduced to assess the quality of the internal models. In cases where internal models lead to a greater number of violations than could reasonably be expected, given the confidence level, the ADI is required to hold a higher level of capital (see Table 1 in the Appendix for the penalties imposed under the Basel II Accord). If an ADI's VaR forecasts are violated more than 10 times in any financial year, the ADI may be required to adopt the 'Standardized' approach. The imposition of such a penalty is severe as it affects the profitability of the ADI directly through higher capital charges, has a damaging effect on the ADI's reputation, and may lead to the imposition of a more stringent external model to forecast the ADI's VaR thresholds. That is why financial managers tend to prefer following strategies that are passive and conservative.

Excessive conservatism has a negative impact on the profitability of ADIs as higher capital charges are subsequently required. Academics and practitioners should ask the question if there is room to minimise the capital charges not only using different models to forecast VaR but also through a communication strategy given the Basel II Accord. ADIs are not allowed to violate more than 10 times in any financial year, but any number less than 10 is permitted. Therefore, the decision maker should seek a strategy 
that allows an endogenous decision as to how many times ADIs should violate in any financial year.

In this paper we characterize a strategic market risk disclosure policy meant to reduce daily capital charges and to manage the number of violations. We suggest that the decision maker should take some actions in each state based on the trade-off between expected capital requirements and the expected number of violations. Financial managers could adopt a different strategy in favourable situations (a small number of violations) than in unfavourable situations (a large number of violations). The amount of expected risk that the manager should report to the monetary authority (namely, a fraction of the VaR estimated using a given procedure) should increase with the number of violations.

In a favourable situation, the decision maker could take more risk (perhaps reporting an expected risk lower than the one suggested by the model used to forecast volatility). In cases of a small number of violations, communication of a low amount of risk allows profiting from the lower capital requirement, subject to having an acceptable trade-off with the upside-risk of increasing the number of violations. If the capital requirement is lower, more funds can be invested in assets at the cost of a marginal increase in the probability of violation. In a situation of a high number of violations, the decision maker must take less risk, and reporting high expected risk (even higher than the forecast) is needed to decrease the probability of violations.

The remainder of the paper is as follows. In Section 2 we present the main ideas of the Basel II Accord Amendment. Section 3 reviews some of the most frequently used univariate $\mathrm{VaR}$ forecasting models. In Section 4 we present the new market risk disclosure strategy. Section 5 gives some experimental results, and Section 6 summarizes the main conclusions.

\section{Forecasting Value-at-Risk and Daily Capital Charges}

The Basel II Accord stipulates that the daily capital charge must be set at the higher of the previous day's VaR or the average VaR over the last 60 business days, multiplied by a factor $k$ : 


$$
C R q_{t}=\operatorname{Max}\left[\operatorname{VaR}(t-1), k \frac{1}{60} \sum_{p=1}^{60} V a \mathrm{R}(t-p)\right] .
$$

The multiplication factor, $k$, is to be set within a range of 3 to 4 depending on the supervisor's assessment of the ADI's risk management practices and on the results of a simple back test (Basel Committee on Banking Supervision (1996)). The multiplication factor is determined by the number of times losses exceed the day's VaR figure (Basel Committee on Banking Supervision (1996)). The minimum multiplication factor of 3 is in place to compensate for a number of errors that arise in model implementation: simplifying assumptions, analytical approximations, small sample biases and numerical errors will tend to reduce the true risk coverage of the model (Stahl (1997)). The increase in the multiplication factor is then designed to scale up the confidence level implied by the observed number of exceptions to the 99 per cent confidence level desired by the regulators.

In calculating the number of exceptions, ADIs will be required to compare the forecasted $\mathrm{VaR}$ numbers with realised profit and loss figures for the previous 250 trading days. In 1995, the 1988 Basel Accord (Basel Committee on Banking Supervision (1988) was amended to allow ADIs to use internal models to determine their VaR thresholds (Basel Committee on Banking Supervision (1995)). However, ADIs wishing to use internal models must demonstrate that their models are sound.

Value-at-Risk refers to the lower bound of a confidence interval for a (conditional) mean. If interest lies in modelling the random variable, $Y_{t}$, it could be decomposed as follows:

$$
Y_{t}=E\left(Y_{t} \mid F_{t-1}\right)+\varepsilon_{t}
$$

This decomposition suggests that $Y_{t}$ is comprised of a predictable component, $E\left(Y_{t} \mid F_{t-1}\right)$, which is the conditional mean, and a random component, $\varepsilon_{t}$. The variability of $Y_{t}$, and hence its distribution, is determined entirely by the variability of $\varepsilon_{t}$. If it is assumed that $\varepsilon_{t}$ follows a distribution such that: 


$$
\varepsilon_{t} \sim D\left(\mu_{t}, \sigma_{t}^{2}\right)
$$

where $\mu_{t}$ and $\sigma_{t}$ are the unconditional mean and standard deviation of $\varepsilon_{t}$, respectively, these can be estimated using a variety of parametric and/or non-parametric methods. The VaR threshold for $Y_{t}$ can be calculated as:

$$
\operatorname{VaR}_{t}=E\left(Y_{t} \mid F_{t-1}\right)-\alpha \sigma_{t}
$$

where $\alpha$ is the critical value from the distribution of $\varepsilon_{t}$ to obtain the appropriate confidence level. It is possible for $\sigma_{t}$ to be replaced by alternative estimates of the conditional variance in order to obtain an appropriate $\mathrm{VaR}$ (for a useful review of recent theoretical results for conditional volatility models, see Li et al. (2002), while McAleer (2005) reviews a variety of univariate and multivariate, conditional, stochastic and realized. volatility models). The next section describes several models that are widely used to forecast the 1-day ahead conditional variances and VaR thresholds.

\section{Models for Forecasting VaR}

As discussed previously, ADIs can use internal models to determine their VaR thresholds. There are alternative time series models for the conditional volatility, $\sigma_{t}$. In what follows, we present several conditional volatility models to evaluate our strategic market risk disclosure, namely GARCH, GJR and EGARCH, with both normal and $t$ distribution errors. For an extensive discussion of the theoretical properties of several of these models, see Ling and McAleer (2002a, 2002b, 2003a). As an alternative to estimating the parameters, we use the exponential weighted moving average (EWMA) method by Riskmetrics ${ }^{T M}$ (1996) that calibrates the unknown parameters. The models are presented in increasing order of complexity.

\subsection{GARCH}

For a wide range of financial data series, time-varying conditional variances can be explained empirically through the autoregressive conditional heteroskedasticity (ARCH) model, which was proposed by Engle (1982). When the time-varying 
conditional variance has both autoregressive and moving average components, this leads to the generalized $\operatorname{ARCH}(p, q)$, or $\operatorname{GARCH}(p, q)$, model of Bollerslev (1986). It is very common to impose the widely estimated $\operatorname{GARCH}(1,1)$ specification in advance.

Consider the stationary AR(1)-GARCH(1,1) model for daily returns, $y_{t}$ :

$$
y_{t}=\phi_{1}+\phi_{2} y_{t-1}+\varepsilon_{t}, \quad\left|\phi_{2}\right|<1
$$

for $t=1, \ldots, n$, where the shocks to returns are given by:

$$
\begin{aligned}
& \varepsilon_{t}=\eta_{t} \sqrt{h_{t}}, \quad \eta_{t} \sim \ddot{i i d}(0,1) \\
& h_{t}=\omega+\alpha \varepsilon_{t-1}^{2}+\beta h_{t-1},
\end{aligned}
$$

and $\omega>0, \alpha \geq 0, \beta \geq 0$ are sufficient conditions to ensure that the conditional variance $h_{t}>0$. The stationary AR(1)-GARCH(1,1) model can be modified to incorporate a nonstationary $\operatorname{ARMA}(\mathrm{p}, \mathrm{q})$ conditional mean and a stationary $\operatorname{GARCH}(\mathrm{r}, \mathrm{s})$ conditional variance, as in Ling and McAleer (2003b).

\subsection{GJR}

In the symmetric GARCH model, the effects of positive shocks (or upward movements in daily returns) on the conditional variance, $h_{t}$, are assumed to be the same as the negative shocks (or downward movements in daily returns). In order to accommodate asymmetric behaviour, Glosten, Jagannathan and Runkle (1992) proposed a model (hereafter GJR), for which GJR $(1,1)$ is defined as follows:

$$
h_{t}=\omega+\left(\alpha+\gamma I\left(\eta_{t-1}\right)\right) \varepsilon_{t-1}^{2}+\beta h_{t-1},
$$

where $\omega>0, \alpha \geq 0, \alpha+\gamma \geq 0, \beta \geq 0$ are sufficient conditions for $h_{t}>0$, and $I\left(\eta_{t}\right)$ is an indicator variable defined by:

$$
I\left(\eta_{t}\right)= \begin{cases}1, & \varepsilon_{t}<0 \\ 0, & \varepsilon_{t} \geq 0\end{cases}
$$


as $\eta_{t}$ has the same sign as $\varepsilon_{t}$. The indicator variable differentiates between positive and negative shocks, so that asymmetric effects in the data are captured by the coefficient $\gamma$. For financial data, it is expected that $\gamma \geq 0$ because negative shocks have a greater impact on risk than do positive shocks of similar magnitude. The asymmetric effect, $\gamma$, measures the contribution of shocks to both short run persistence, $\alpha+\gamma / 2$, and to long run persistence, $\alpha+\beta+\gamma / 2$.

\subsection{EGARCH}

An alternative model to capture asymmetric behaviour in the conditional variance is the Exponential GARCH, EGARCH(1,1), model of Nelson (1991), namely:

$$
\log h_{t}=\omega+\alpha\left|\frac{\varepsilon_{t-1}}{h_{t-1}}\right|+\gamma \frac{\varepsilon_{t-1}}{h_{t-1}}+\beta \log h_{t-1}, \quad|\beta|<1
$$

where the parameters $\alpha, \beta$ and $\gamma$ have different interpretations from those in the $\operatorname{GARCH}(1,1)$ and $\operatorname{GJR}(1,1)$ models.

As noted in McAleer et al. (2007), there are some important differences between EGARCH and the previous two models, as follows: (i) EGARCH is a model of the logarithm of the conditional variance, which implies that no restrictions on the parameters are required to ensure $h_{t}>0$; (ii) moment conditions are required for the GARCH and GJR models as they are dependent on lagged unconditional shocks, whereas EGARCH does not require moment conditions to be established as it depends on lagged conditional shocks (or standardized residuals);

EGARCH captures asymmetries differently from GJR. The parameters $\alpha$ and $\gamma$ in EGARCH $(1,1)$ represent the magnitude (or size) and sign effects of the standardized residuals, respectively, on the conditional variance, whereas $\alpha$ and $\alpha+\gamma$ represent the effects of positive and negative shocks, respectively, on the conditional variance in $\operatorname{GJR}(1,1)$. 


\subsection{Exponentially Weighted Moving Average (EWMA)}

The three conditional volatility models given above are estimated under the following distributional assumptions on the conditional shocks: (1) normal, and (2) t. As an alternative to estimating the parameters of the appropriate conditional volatility models, Riskmetrics $^{T M}$ (1996) developed a model which estimates the conditional variances and covariances based on the exponentially weighted moving average (EWMA) method, which is, in effect, a restricted version of the $\mathrm{ARCH}(\infty)$ model. This approach forecasts the conditional variance at time $t$ as a linear combination of the lagged conditional variance and the squared unconditional shock at time $t-1$. The EWMA model calibrates the conditional variance as:

$$
h_{t}=\lambda h_{t-1}+(1-\lambda) \varepsilon_{t-1}^{2}
$$

where $\lambda$ is a decay parameter. Riskmetrics ${ }^{\mathrm{TM}}$ (1996) suggests that $\lambda$ should be set at 0.94 for purposes of analysing daily data. As no parameters are estimated, there is no need to establish any moment or log-moment conditions for purposes of demonstrating the statistical properties of the estimators.

\section{A Dynamic Decision Rule for Strategic Market Risk Disclosure}

Recent empirical studies (see, for example, Berkowitz and O'Brien (2001) and Gizycki and Hereford (1998)) indicate that some financial institutions overestimate their market risks in disclosures to supervisory authorities. This implies a costly restriction to the banks trading activity. ADIs may prefer to report high VaR numbers to avoid the possibility of regulatory intrusion. This conservative risk reporting suggests that efficiency gains may be feasible. Therefore, as ADIs already have effective tools for the measurement of market risk, while satisfying the qualitative requirements, ADI managers could wilfully attempt to reduce the daily capital charges by implementing a context-dependent market risk disclosure policy. For a discussion of alternative approaches to optimize VaR and daily capital charges, see McAleer (2008).

The novel feature of our analysis is the assumption that ADIs may minimize daily capital charges while the number of violations remains below 10. In particular, we 
assume market risk disclosures should be proportional to the VaR measured based on the ADI's internal model. The intuition is that the ADI manager is willing to be conservative when the number of violations could be close to ten, and aggressive when the number of violations is small. We find that when the ADI manager uses our dynamic rule decision to market risk disclosures, taking into account the number of violations, the average daily capital charges during the 260 trading days of 2007 can decrease by up to $14 \%$. A special case of the endogenous violations discussed above, in which the number of violations is not a choice variable but is exogenously determined, is analysed in McAleer and da Veiga (2008a, 2008b).

In this paper we propose a simple market risk disclosure policy based on a new dynamic decision rule designed to minimize daily capital charges while restricting the number of violations to less than 10 . The question here is what proportion of the VaR calculated by the ADI's internal model should be reported as a market risk measure. If we follow a conservative risk reporting strategy (that is, a market risk disclosure greater than the VaR), we will commit few violations but will incur higher capital costs. However, if the market risk disclosure is smaller than the VaR, we would have a relatively low capital requirement but a high risk of incurring too many violations.

We propose the following Market Risk Disclosure (MRD) Policy:

$$
M R D_{t}=P_{t} \operatorname{VaR}_{t}
$$

where $P_{t}$ varies with the number of violations to communicate risk to the monetary authority. The variable $P_{t}$ is a measure of how conservative or aggressive the MRD is in comparison with the estimated risk: $P_{t}<1$ corresponds to an aggressive strategy because the MRD is below the estimated risk, whereas $P_{t}>1$ represents a conservative approach.

\section{Dynamic Learning Strategy (DYLES)}

$$
P_{t}=P_{0}+\theta^{P} * n o v_{t-1}-\theta^{R} \sum_{s=1}^{t} I_{25, s}
$$


The dynamic learning function, $P_{t}$, consists of three additive terms:

(1) $P_{0}$ is an initial condition and, as time passes, has a decreasing effect on DYLES.

(2) $\theta^{P}$ is the penalty for each violation: any additional violation should be penalized, thereby increasing the market risk disclosure and making our strategy more conservative.

(3) $n o v_{t-1}$ is the number of violations up to period $t-1$.

(4) $\theta^{R}$ is the reward (that is, the reduction in the penalty) for each 25 -day period without any violations.

(5) $\mathrm{I}_{25, \mathrm{t}}$ indicates whether there has been a violation in a given period. We divide the 250-day testing period, into 10 fixed periods of 25 days. At the end of each 25-day interval, we check whether there have been any violations during the period. If there have been no violations, the reward consists of decreasing the penalty by $\theta^{R}$.

The indicator function, $I_{25, t}$, performs the counting: it takes the value one when there have been no violations during a fixed 25-day period, and zero otherwise. It can only change value at the end of each 25-day period. At that point, the reward is either given or it is not:

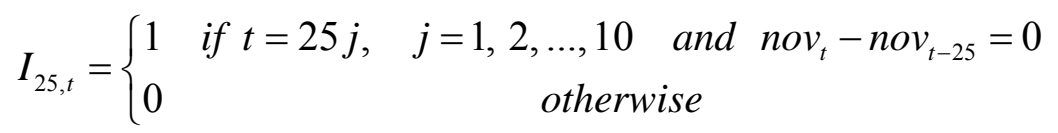

This dynamic learning penalty function, $P_{t}$, is designed to decrease the capital requirements of a MRD policy based on a given $\mathrm{VaR}$, while restricting the number of violations in a given period within the limits of the Basel II Accord.

DYLES works in a complementary manner with the volatility model, but behaves quite differently from the volatility models: 
- It operates in a discrete and fast manner when there is a violation, whereas the volatility models adjust smoothly to violations.

- It is context sensitive as it takes different values depending on the history of the violations. It is more conservative when there have been many violations, and is less conservative when there have been fewer violations.

- It operates asymmetrically over time, as it reacts immediately when there is a violation, but moves discretely when there is a period without any violations.

The parameters of the penalty function would need to be calibrated for a given asset and for each model to calculate VaR. In the next section, we provide some insights as to how well this function works for a given portfolio and for different models of the conditional variance.

\section{Experimental Results}

When we have proposed a new MRD policy based on a dynamic learning penalty function, we are interested in assessing how well it performs in terms of daily capital costs and the number of violations compared with the alternative strategy (that is, no strategy) of not responding to either violations or the absence of violations.

Owing to the dearth of theoretical results in this area, we examine the behaviour of our penalty function using calibration. As the basis for comparison we use Standard and Poor's Composite 500 index from 1 January 2007 to 31 December of 2007. The parameters in the vector $\Theta=\left[P_{0}>0, \theta^{P}>0, \theta^{R}>0\right]$ in (12) have to be positive, and calibration suggests the following intervals:

$$
\begin{aligned}
& P_{0} \in[0.6, \ldots, 1.2], \\
& \theta^{P} \in[0.06, \ldots, 0.12], \\
& \theta^{R} \in[0.1, \ldots, 0.4] .
\end{aligned}
$$


$\mathrm{P}_{0}<0.6$ (aggressive strategies) would imply numbers of violations in excess of 10 , and $\mathrm{P}_{0}$ greater than 1.2 (conservative risk reporting) would lead to high daily capital requirements.

The calibration procedure is as follows

1. We assume the models described in Section 3 to be the internal ADI's models that are used to forecast the 1-day ahead conditional variances and VaR thresholds.

2. For all possible parameter combinations of $\Theta$, we calculate $P_{t}$, as given in (12).

3. Given the VaR calculated in step 1 and $P_{t}$ in step 2, calculate the market risk disclosure using (11).

4. The number of violations (NoV) and the average capital requirements (AvCRq) for the whole period are reported.

5. Finally, we compare $\mathrm{NoV}$ and the AvCRq requirements with the no strategy policy.

\subsection{Data and volatility measures}

The data used for the calibration of DYLES are the closing daily prices for Standard and Poor's Composite 500 Index. Data were obtained from the Ecowin Financial Database for the period 3 January 2000 to 31 December 2007.

The returns at time $t\left(R_{t}\right)$ are defined as:

$$
R_{t}=\log \left(P_{t} / P_{t-1}\right)
$$

where $P_{t}$ is the market price.

[Insert Figure 1 around here]

Figure 1 shows the Standard and Poor's returns. The series exhibit clustering, which could be captured by an appropriate time series model. The descriptive statistics for the index returns are given in Table 2. The mean is close to zero, and the range is between - 
$6 \%$ and $5.57 \%$. The Jarque-Bera Lagrange multiplier test for normality rejects the null hypothesis of normally distributed returns. As the series displays a high kurtosis, this would seem to indicate the existence of extreme observations, which is not surprising for financial returns data.

\section{[Insert Table 2 around here]}

Several measures of volatility are available in the literature. In order to gain some intuition, we adopt the measure proposed in Franses and van Dijk (1999), where the true volatility of returns is defined as:

$$
V_{t}=\left(R_{t}-E\left(R_{t} \mid F_{t-1}\right)\right)^{2}
$$

where $F_{t-1}$ is the information set at time $t-1$. Figure 2 shows the S\&P volatility defined as in (15). The series exhibit clustering, which needs to be captured by an appropriate time series model. The volatility of the series appears to be high during the early 2000's, followed by a quiet period from 2003 to the beginning of 2007. The volatility appears to increase dramatically around 2007, due in large part to the worsening global credit environment. This increase in volatility persists until the end of the period, and continues during 2008.

[Insert Figure 2 around here]

[Insert Table 3 around here]

\subsection{Results}

In Table 3 we see the differences between using DYLES or a passive strategy for the S\&P and several VaR models. After calibration, we have chosen the set of parameters $\mathrm{P}_{0}=1.2, \theta^{\mathrm{P}}=0.12, \theta^{\mathrm{R}}=0.3$, which is the combination which seems to be most widely optimal across the various models. 
Figure 1

S\&P_RETURNS

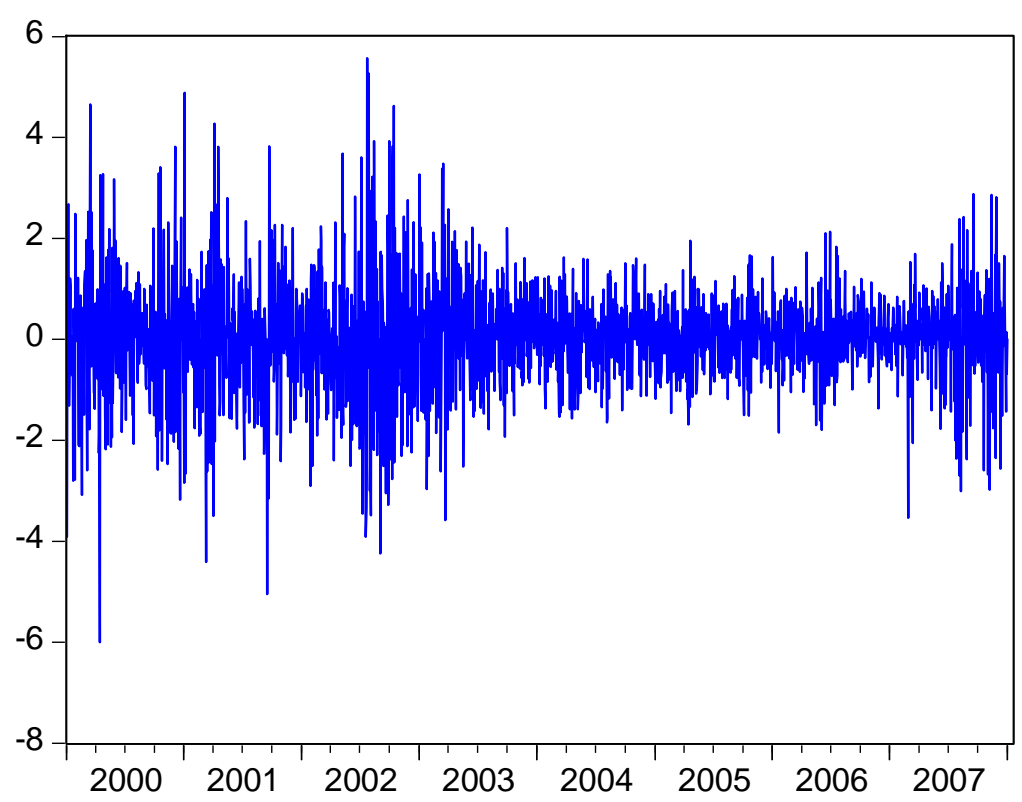


Table 2

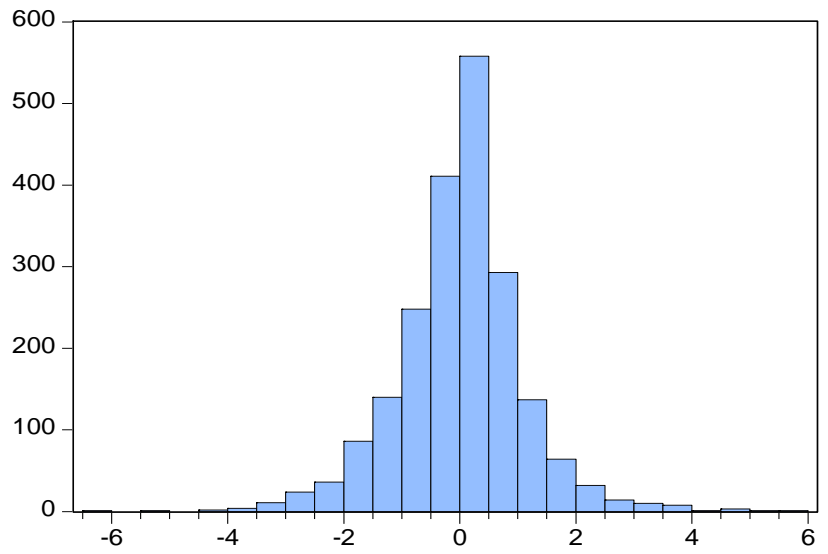

Series: SP_RETURNS

Sample 3/01/2000 18/01/2008

Observations 2086

Mean

Median

0.000431

Minimum $\quad-6.004513$

Std. Dev. $\quad 1.094369$

Skewness $\quad 0.048622$

Kurtosis $\quad 5.760783$

Jarque-Bera $\quad 663.2940$

Probability $\quad 0.000000$ 
We observe in the last column that the capital requirement decreases in all cases between $0.4 \%$ (3 basis points) and 14.01\% (96 basis points) when using DYLES, except when the GARCH-t model is used. Moreover, the number of violations decreases when they were above 10 and increases, within the limits, when there are few violations. The exception in Table 4 in the Appendix shows that the best result for the GARCH-t model and DYLES is for $\Theta=[1.0,0.11,0.3]$ with 9 violations and $5.80 \%$ as AvCRq, that is, 90 basis points less than the no strategy policy.

We conclude that DYLES always beats the passive strategy when properly calibrated. Due to its context-sensitive behaviour, DYLES tends to concentrate the distribution of the number of violations. In cases where there is conservative behaviour, it tends to increase the number of violations, whereas when the number of violations is large, it tends to reduce it to below the limit of 10 .

When properly used, DYLES can decrease the daily capital requirements substantially, up to 96 basis points in the case analyzed above, while restricting the number of violations to within the limits of the Basel II Accord.

\section{[Insert Figure 3 around here]}

In order to gain some intuition, in Figure 3 we present a comparison of DYLES with the results for Riskmetrics ${ }^{T M}$.

a. The returns data are for the Standard and Poor's index during the last 100 days of 2007.

b. The stepwise line corresponds to the values of DYLES $\left(P_{t}\right)$ during the period for which $\Theta=[1.2,0.12,0.3]$.

c. Of the two bottom lines, the one that starts higher is the VaR calculated by Riskmetrics $^{T M}$

d. Of the two bottom lines, the one (line with symbols) that starts lower (with greater risk) is the VaR of Riskmetrics ${ }^{T M}+$ DYLES, which is our RMD. 
Figure 2

S\&P Volatility

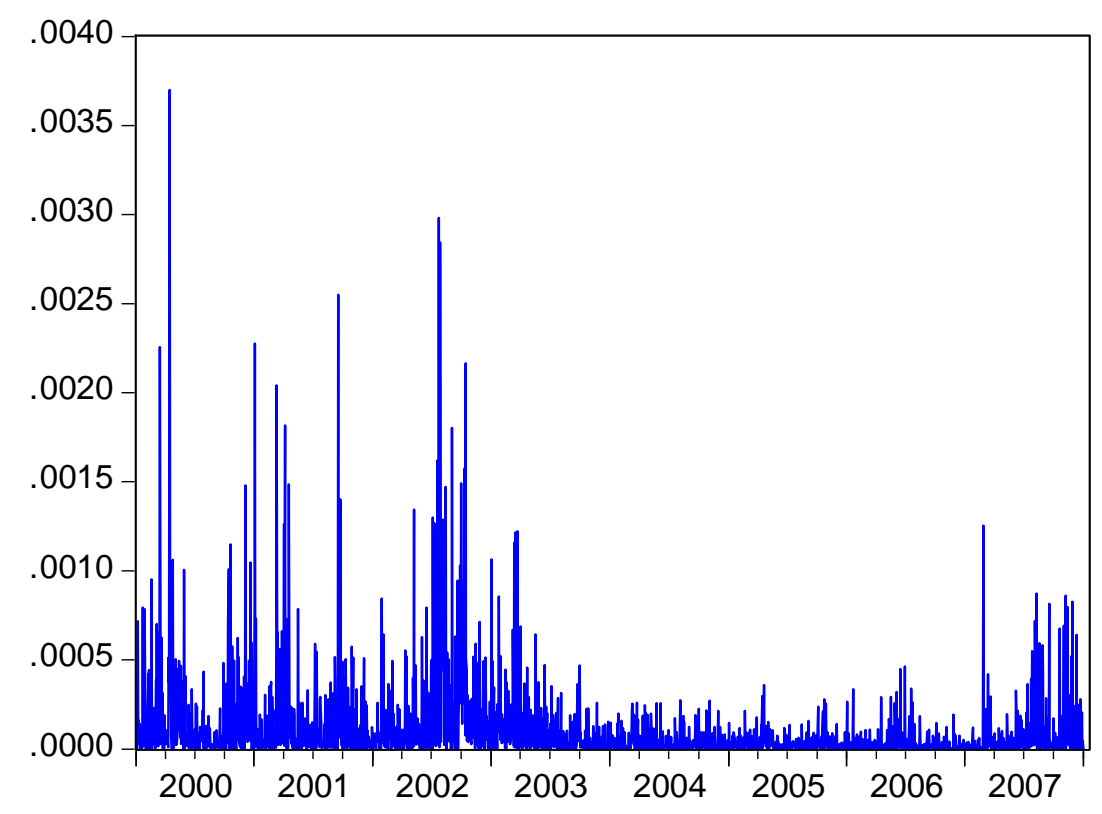


Table 3

Benefits from DYLES, Standard and Poor's, $\theta=[1.2,0.12,0.3]$

\begin{tabular}{|c|c|c|c|c|c|c|c|}
\hline \multirow{2}{*}{ Model } & \multicolumn{2}{|c|}{ DYLES } & \multicolumn{2}{c|}{ Passive } & \multicolumn{3}{c|}{ Improvement } \\
& \multicolumn{2}{|c|}{ DYLES vs Passive } \\
\hline & NoV & AvCRq & NoV & AvCRq & NoV & AvCRq & Basic points \\
\hline EGARCH & 9 & $5.94 \%$ & 13 & $6.24 \%$ & -4 & $4.8 \%$ & 30 \\
\hline EGARCH-t & 7 & $5.86 \%$ & 9 & $6.82 \%$ & -2 & $14.1 \%$ & 96 \\
\hline GARCH & 8 & $6.00 \%$ & 11 & $6.54 \%$ & -3 & $8.3 \%$ & 54 \\
\hline GARCH-t & 7 & $7.00 \%$ & 3 & $6.70 \%$ & 4 & $-4.5 \%$ & -30 \\
\hline GJR & 8 & $6.00 \%$ & 9 & $6.75 \%$ & -1 & $11.1 \%$ & 75 \\
\hline GJR-t & 8 & $7.01 \%$ & 4 & $7.04 \%$ & 4 & $0.4 \%$ & 3 \\
\hline Riskmetrics ${ }^{T M}$ & 8 & $5.98 \%$ & 12 & $6.61 \%$ & -4 & $9.5 \%$ & 63 \\
\hline
\end{tabular}


Note that when the Riskmetrics ${ }^{T M}+$ DYLES line is above the Riskmetrics ${ }^{T M}$ line, it implies less risk and leads to lower daily capital requirements. The improvement in capital requirements is given by the difference between the red (dark) and blue (light) lines in Figure 3. This is attributable exclusively to the penalty function of the dynamic learning function strategy (DYLES).

Figure 4 shows the four previous series for the whole sample, from 1 January 2007 to 31 December 2007. As we can see, the lines representing the Riskmetrics ${ }^{T M} \mathrm{VaR}$ and the RMD policy move together when there is no violation. As soon as a violation occurs, the DYLES strategy becomes more conservative and moves away from zero until a period of no violations occurs, when it becomes more aggressive and moves toward zero.

[Insert Figure 4 around here]

Overall, the capital requirements for DYLES are lower than when DYLES is not used by $9.5 \%$ (63 basis points). The number of violations also decreases from 12 to 8 . This is a general pattern observed across different VaR models to varying degrees, as seen in Table 3 above.

This example suggests that, with proper calibration, the DYLES strategy can help decrease daily capital requirements while restricting the number of violations to within the desired limits.

Tables 4-7 in the Appendix show the NoV and AvCRq for values of $\Theta$ when two conditions are met: (i) NoV is less than 10; and (ii) AvCRq is less than $6.00 \%$. The first criterion is consideredbecause we want our MRD to be sound for the monetary authority, while the second considers a reduction in the AvCRq by 9\% (61 basis points) when compared with the Riskmetrics ${ }^{T M}$ procedure. 


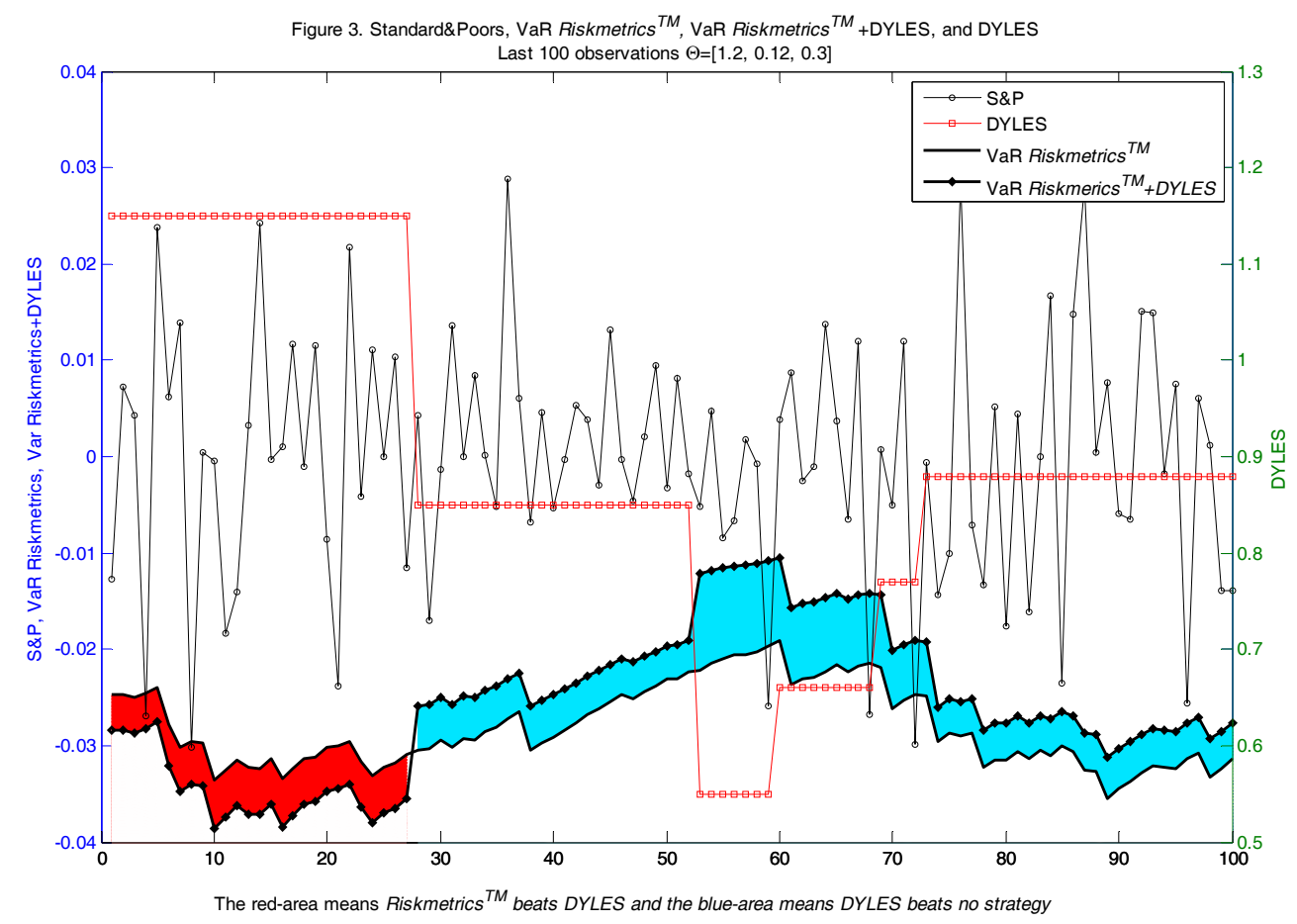


Figure 4

Standard\&Poor's / VaR Riskmetrics ${ }^{T M}$ / VaR Riskmetrics ${ }^{T M}+$ DYLES / Dyles 260 observations. $\Theta=[1.2,0.12,0.3]$.

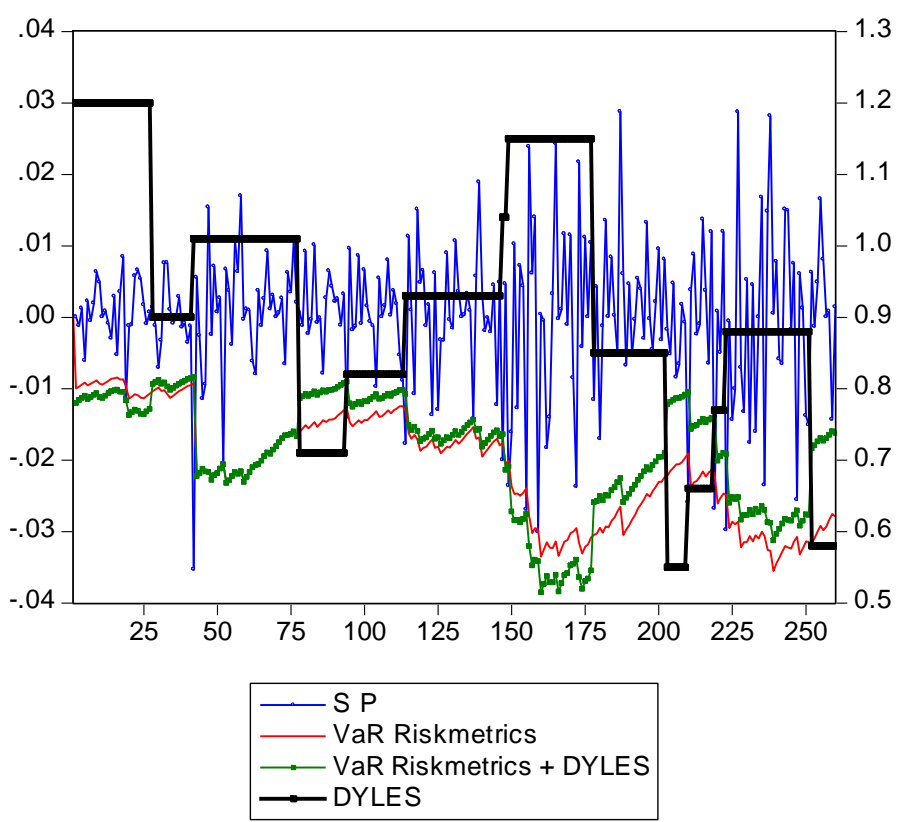


Based on Tables 4-7 and the previous analysis, we conclude that there are combinations of parameters in $\Theta$ that can reduce the daily capital requirements compared with existing models and strategies, while producing an acceptable number of violations. It would seem to be straightforward to find a parameter vector $\Theta$ with a systematically smaller AvCRq and NoV below 10 for all the models, when compared with the no strategy policy.

It is noteworthy that, while $\mathrm{VaR}$ is used by numerous financial institutions, it is not without shortcomings. The VaR measure can under or overestimate risk. There is even debate as to how best to model the behaviour of volatility in market returns. Relying on DYLES, which modulates market risk disclosure, can reduce the effects of these deficiencies, as DYLES concentrates NoV throughout the models tested. In some of the cases discussed above, DYLES can control the number of violations at low cost in terms of the daily capital requirements.

\section{Conclusion}

Under the Basel II Accord, ADIs have to communicate their risk estimates to the monetary authorities, and use a variety of VaR models to estimate risks. ADIs are subject to a back-test that compares the daily VaR to the subsequent realized returns, and ADIs that fail the back-test can be subject to the imposition of standard models that can lead to higher daily capital costs. Additionally, the Basel II Accord stipulates that the daily capital charge that the bank must carry as protection against market risk must be set at the higher of the previous day's VaR or the average VaR over the last 60 business days, multiplied by a factor $k$. An ADI's objective is to maximize profits, so they wish to minimize their capital charges while restricting the number of violations in a given year below the maximum of 10 allowed by the Basel II Accord.

VaR models currently in use can lead to high daily capital requirements or an excessive number of violations. In this paper we proposed a new dynamic learning strategy, DYLES, designed to minimize the daily capital requirements, while restricting the number of violations to below the penalty limit. We designed a market risk disclosure strategy driven by the number of violations to communicate the risk to the monetary authority. The strategy is context sensitive, and depends on the history of violations. It 
is intended to penalize $\mathrm{VaR}$ models when a loss exceeds the reported VaR by increasing the risk for the following periods. On the other hand, after a given period with no violations, the criterion offers a reward by decreasing the reported risk.

In order to illustrate the practicability of DYLES, we applied it to the Standard and Poor 500 Index using seven different VaR models. After estimation of the VaR models and calibration of the parameters, we showed that it could lower the daily capital requirements substantially (by up to $14.3 \%$, or 95 basis points, when we compared, for example, the GJR- $t$ model + DYLES to the no strategy Riskmetrics ${ }^{T M}$ policy), while restricting the numbers of violations to within the Basel II Accord limits ( 9 for GJR- $t+$ DYLES and 12 for the no strategy Riskmetrics ${ }^{T M}$ policy ).

Simplicity would seem to have been the key to the popularity of VaR, particularly as a means of providing information to an ADI's senior management. DYLES is as simple and intuitive as $\mathrm{VaR}$. When there is a violation, it increases immediately, thereby becoming more conservative and decreasing the risks of further violations, whereas after a period of no violations, it becomes less conservative, thereby allowing lower daily capital requirements.

The preceding arguments suggest that DYLES can be used profitably by ADIs to reduce their average daily capital requirements, while restricting the numbers of violations to the Basel II Accord penalty limits. 


\section{References}

Basel Committee on Banking Supervision, (1988), International Convergence of Capital Measurement and Capital Standards, BIS, Basel, Switzerland.

Basel Committee on Banking Supervision, (1995), An Internal Model-Based Approach to Market Risk Capital Requirements, BIS, Basel, Switzerland.

Basel Committee on Banking Supervision, (1996), Supervisory Framework for the Use of "Backtesting" in Conjunction with the Internal Model-Based Approach to Market Risk Capital Requirements, BIS, Basel, Switzerland.

Berkowitz, J. and J. O'Brien (2001), How accurate are value-at-risk models at commercial banks?, Discussion Paper, Federal Reserve Board.

Bollerslev, T. (1986), Generalised autoregressive conditional heteroscedasticity, Journal of Econometrics, 31, 307-327.

Engle, R.F. (1982), Autoregressive conditional heteroscedasticity with estimates of the variance of United Kingdom inflation, Econometrica, 50, 987-1007.

Franses, P.H. and D. van Dijk (1999), Nonlinear Time Series Models in Empirical Finance, Cambridge, Cambridge University Press.

Gizycki, M. and N. Hereford (1998), Assessing the dispersion in banks' estimates of market risk: the results of a value-at-risk survey, Discussion Paper 1, Australian Prudential Regulation Authority.

Glosten, L., R. Jagannathan and D. Runkle (1992), On the relation between the expected value and volatility of nominal excess return on stocks, Journal of Finance, 46, 1779-1801.

Jorion, P. (2000), Value at Risk: The New Benchmark for Managing Financial Risk, McGraw-Hill, New York.

Li, W.K., S. Ling and M. McAleer (2002), Recent theoretical results for time series models with GARCH errors, Journal of Economic Surveys, 16, 245-269. Reprinted in M. McAleer and L. Oxley (eds.), Contributions to Financial Econometrics: Theoretical and Practical Issues, Blackwell, Oxford, 2002, pp. 933.

Ling, S. and M. McAleer (2002a), Stationarity and the existence of moments of a family of GARCH processes, Journal of Econometrics, 106, 109-117. 
Ling, S. and M. McAleer (2002b), Necessary and sufficient moment conditions for the GARCH(r,s) and asymmetric power GARCH(r, s) models, Econometric Theory, $18,722-729$.

Ling, S. and M. McAleer, (2003a), Asymptotic theory for a vector ARMA-GARCH model, Econometric Theory, 19, 278-308.

Ling, S. and M. McAleer (2003b), On adaptive estimation in nonstationary ARMA models with GARCH errors, Annals of Statistics, 31, 642-674.

McAleer, M. (2005), Automated inference and learning in modeling financial volatility, Econometric Theory, 21, 232-261.

McAleer, M. (2008), The Ten Commandments for optimizing value-at-risk and daily capital charges, to appear in Journal of Economic Surveys.

McAleer, M., F. Chan and D. Marinova (2007), An econometric analysis of asymmetric volatility: theory and application to patents, Journal of Econometrics, 139, 259284.

McAleer, M. and B. da Veiga (2008a), Forecasting value-at-risk with a parsimonious portfolio spillover GARCH (PS-GARCH) model, Journal of Forecasting, 27, 1-19.

McAleer, M. and B. da Veiga (2008b), Single index and portfolio models for forecasting value-at-risk thresholds, Journal of Forecasting, 27, 217-235.

Nelson, D.B. (1991), Conditional heteroscedasticity in asset returns: a new approach, Econometrica, 59, 347-370.

Riskmetrics $^{\mathrm{TM}}$ (1996), J.P. Morgan Technical Document, $4^{\text {th }}$ Edition, New York, J.P. Morgan.

Stahl, G. (1997), Three cheers, Risk, 10, 67-69. 


\section{Appendix 1}

Table 1: Basel Accord Penalty Zones

\begin{tabular}{|l|l|l|}
\hline Zone & Number of Violations & Increase in k \\
\hline Green & 0 to 4 & 0.00 \\
\hline Yellow & 5 & 0.40 \\
& 6 & 0.50 \\
& 8 & 0.65 \\
& 9 & 0.75 \\
\hline Red & $10+$ & 0.85 \\
\hline \\
$\begin{array}{l}\text { Note: The number of violations is given for } 250 \text { business days. } \\
\text { The penalty structure under the Basel II Accord is specified for } \\
\text { the number of penalties and not their magnitude, either } \\
\text { individually or cumulatively. }\end{array}$ \\
\hline
\end{tabular}


Table 4

MRD results when NoV $<=10$ and $\mathrm{CRq}<6.1 \%$

\begin{tabular}{|c|c|c|c|c|c|c|c|c|c|}
\hline \multicolumn{5}{|c|}{ EGARCH } & \multicolumn{7}{|c|}{ EGARCH-t } \\
\hline NoV & AvCRq & $\mathbf{P}_{\mathbf{0}}$ & $\boldsymbol{\theta}^{\mathbf{P}}$ & $\boldsymbol{\theta}^{\mathbf{R}}$ & $\mathbf{N o V}$ & AvCRq & $\mathbf{P}_{\mathbf{0}}$ & $\theta^{\mathbf{P}}$ & $\boldsymbol{\theta}^{\mathbf{R}}$ \\
\hline 7 & $5.97 \%$ & 0.9 & 0.10 & 0.1 & 9 & $5.91 \%$ & 0.8 & 0.11 & 0.3 \\
\hline 9 & $5.91 \%$ & 0.9 & 0.12 & 0.3 & 9 & $5.94 \%$ & 1.1 & 0.06 & 0.2 \\
\hline 9 & $6.00 \%$ & 1.0 & 0.10 & 0.3 & 9 & $5.95 \%$ & 1.1 & 0.11 & 0.3 \\
\hline 9 & $5.70 \%$ & 1.2 & 0.06 & 0.2 & 9 & $5.87 \%$ & 1.2 & 0.09 & 0.3 \\
\hline 9 & $5.91 \%$ & 1.2 & 0.07 & 0.2 & 8 & $5.70 \%$ & 1.2 & 0.11 & 0.3 \\
\hline 7 & $5.93 \%$ & 1.2 & 0.10 & 0.2 & 7 & $5.86 \%$ & 1.2 & 0.12 & 0.3 \\
\hline 9 & $5.94 \%$ & 1.2 & 0.12 & 0.3 & & & & & \\
\hline
\end{tabular}

Table 5

MRD results when NoV $<=10$ and $\mathrm{CRq}<6.1 \%$

\begin{tabular}{|c|c|c|c|c|c|c|c|c|c|}
\hline \multicolumn{9}{|c|}{ GARCH } & \multicolumn{7}{c|}{ GARCH-t } \\
\hline NoV & AvCRq & $\mathbf{P}_{\mathbf{0}}$ & $\boldsymbol{\theta}^{\mathbf{P}}$ & $\boldsymbol{\theta}^{\mathbf{R}}$ & $\mathbf{N o V}$ & AvCRq & $\mathbf{P}_{\mathbf{0}}$ & $\boldsymbol{\theta}^{\mathbf{P}}$ & $\boldsymbol{\theta}^{\mathbf{R}}$ \\
\hline & & & & & & & & & \\
9 & $5.89 \%$ & 0.8 & 0.11 & 0.3 & 7 & $6.00 \%$ & 0.9 & 0.11 & 0.2 \\
\hline 9 & $5.93 \%$ & 1.1 & 0.11 & 0.3 & 9 & $5.80 \%$ & 1.0 & 0.11 & 0.3 \\
\hline & & & & & & & & & \\
8 & $5.85 \%$ & 1.2 & 0.11 & 0.3 & 8 & $5.87 \%$ & 1.1 & 0.11 & 0.3 \\
\hline
\end{tabular}


Table 6

MRD results when NoV $<=10$ and $\mathrm{CRq}<6.1 \%$

\begin{tabular}{|c|c|c|c|c|c|c|c|c|c|}
\hline \multicolumn{9}{|c|}{ GJR } & \multicolumn{7}{c|}{ GJR-t } \\
\hline NoV & AvCRq & $\mathbf{P}_{\mathbf{0}}$ & $\boldsymbol{\theta}^{\mathbf{P}}$ & $\boldsymbol{\theta}^{\mathbf{R}}$ & $\mathbf{N o V}$ & AvCRq & $\mathbf{P}_{\mathbf{0}}$ & $\boldsymbol{\theta}^{\mathbf{P}}$ & $\boldsymbol{\theta}^{\mathbf{R}}$ \\
\hline 9 & $5.85 \%$ & 0.6 & 0.11 & 0.2 & 8 & $6.00 \%$ & 0.6 & 0.10 & 0.2 \\
\hline 9 & $5.92 \%$ & 1.1 & 0.06 & 0.2 & 8 & $5.90 \%$ & 1.0 & 0.07 & 0.2 \\
\hline 9 & $5.92 \%$ & 1.1 & 0.12 & 0.3 & 9 & $5.66 \%$ & 1.0 & 0.12 & 0.3 \\
\hline 9 & $5.87 \%$ & 1.2 & 0.11 & 0.3 & 9 & $5.90 \%$ & 1.1 & 0.10 & 0.3 \\
\hline
\end{tabular}

Table 7

MRD results when NoV $<=10$ and $\mathrm{CRq}<6.1 \%$

\begin{tabular}{|c|c|c|c|c|}
\hline \multicolumn{5}{|c|}{ Riskmetrics $^{\text {TM }}$} \\
\hline NoV & AvCRq & P $_{\mathbf{0}}$ & $\theta^{\mathbf{P}}$ & $\theta^{\mathbf{R}}$ \\
\hline & & & & \\
9 & $5.81 \%$ & 0.8 & 0.11 & 0.3 \\
\hline & & & & \\
\hline 8 & $5.76 \%$ & 1.2 & 0.11 & 0.3 \\
\hline 8 & & & & \\
\hline
\end{tabular}

